\title{
Simulation of vortex cores switching in nanocolumnar conducting triplex structure
}

\author{
A.E. Ekomasov ${ }^{\dagger}$, S.V. Stepanov, E.G. Ekomasov \\ †ekomasovae@gmail.com
}

Bashkir State University, Z. Validi 32, 450076, Ufa, Russia

\begin{abstract}
With the generalized Landau-Lifshitz equation the dynamics of the magnetization in the permalloy nanopillars of small diameter of $120 \mathrm{~nm}$ is studied. For the numerical calculation of the magnetic vortices bound dynamics a software package for micromagnetic simulations SpinPM was used. The nanopillars have two magnetic layers separated by a nonmagnetic layer. The study of two coupled magnetic vortices dynamics under the influence of an external magnetic field perpendicular to the plane of the sample and polarized electric current was conducted. The coupled magnetic vortices were taken with the same polarity and chirality. By using micromagnetic simulation the dependence of the magnetic field, switching the polarity of the vortex core in thin and thick layers, on the current strength was found. For the case of low currents, the vortex polarity switch in thin and thick layers was observed with a low exit of the vortex from the geometric center. For the case of high currents, the dynamic mechanism for the vortex core polarity switching was observed for a vortex in a thick layer for all values of the current polarization. For the vortex in a thin layer the dynamic mechanism of the vortex core polarity switching was observed only for the case of a large polarization. The minimum current value in the high current region (similarly to the case of nanocylinder with a diameter of $200 \mathrm{~nm}$ ) significantly decreases with the increase of the current polarization value.
\end{abstract}

Keywords: generalized Landau-Lifshitz equation, magnetic vortices, nonlinear dynamics, magnetic nanodiscs.

\section{Introduction}

The researchers demonstrate great interest in permalloy nanopillars that have two magnetic layers separated by a nonmagnetic interlayer [1]. The magnetic vortex can be realized in them as a basic state. There are two possible directions of magnetization in the vortex core (or core polarity) - up or down, and that can be used, for example, in digital devices. However large energy density in the core leads to the presence of a large potential barrier for the core polarity switching. The static properties and structure of the magnetic vortices in nanodiscs are well studied (e.g., vortex state stability as a function of system parameters and external influences) $[1,2]$. It was also shown that by passing a spinpolarized current through the magnetic disk, the vortex can start moving along orbits of different types (for example, along a circular stationary orbit, chaotic, etc.).) [2, 3]. The mechanism of the magnetic vortex polarity switching, as polarized by current and by pulsed or constant magnetic field, was also covered. For the core polarity switching with a large, perpendicular to the disc plane, static external magnetic field a "static" switching mechanism has been proposed [4]. The suggested later "dynamic" scenario [5] (in the presence of a spin-polarized current) shows that one can substantially reduce the magnetic switch field. In the "dynamic" scenario the vortex core goes out of the center and moves along the increasing orbit simultaneously increasing the speed. Near the vortex core a pair vortex is formed (of opposite polarity) - antivortex. Finally, when the speed reaches a critical point, the initial vortex and antivortex are destroyed, and the remaining vortex of opposite polarity begins to move in the opposite direction along the decreasing orbit.

There are many experimental and theoretical papers devoted to the study of the magnetostatically bound magnetic vortices dynamics. For example, the dependence of the magnetic field size, switching all vortices polarities, on the polarized current magnitude for nanodisks of different diameters was experimentally found [6]. It is shown that for a system of two interacting magnetic disks in the vortex state, the magnetic vortices oscillation spectrum can change fundamentally [7]. In this paper, we theoretically investigate the influence of the perpendicular magnetic field, size and degree of current polarization on the bound vortices dynamics in nanodisks with a diameter of $120 \mathrm{~nm}$.

\section{Basic equations and results}

The nonlinear dynamics of magnetization in a magnetic layer is described by the generalized Landau-Lifshitz equation (GLLE) [8]. It contains additional torque $\vec{T}_{s, t}$, responsible for the interaction of current with magnetization and has the form:

$$
\dot{\overrightarrow{\mathbf{M}}}=-\gamma\left[\overrightarrow{\mathbf{M}} \times \overrightarrow{\mathbf{H}}_{\text {eff }}\right]+\frac{\alpha}{M_{s}}[\overrightarrow{\mathbf{M}} \times \dot{\overrightarrow{\mathbf{M}}}]+\vec{T}_{\text {s.t. }}
$$

where $\overrightarrow{\mathbf{M}}$ - magnetization vector, $\gamma$ - gyromagnetic ratio, $M_{s}$ - saturation magnetization, $\alpha-$ Gilbert damping parameter. The effective field $\overrightarrow{\mathbf{H}}_{\text {eff }}$ represents a sum of an external magnetic field, magnetostatic and exchange interaction fields. 


$$
\begin{aligned}
& T_{\text {s.t. }}=-\frac{\gamma_{0} a_{J}}{M_{s}} \mathbf{M} \times\left[\mathbf{M} \times \mathbf{m}_{r e f}\right]+\gamma b_{J} \mathbf{M} \times \mathbf{m}_{r e f} \\
& a_{j}=\frac{\hbar}{2|e|} \frac{1}{d} P \frac{1}{M_{s}} J_{e}, \quad b_{j}=\beta a_{j}, \quad \beta \sim 0.05-0.2
\end{aligned}
$$

Here, $a_{j}, b_{j}$ are the coefficients proportional to the current density $J_{e}$, having the dimensionality of the field and depending on the material parameters and characteristics of the multilayer structure boundaries. $\mathbf{m}_{\text {ref }}$ is a unity vector along the magnetization of the support layer. GLLE is a complex integro-differential equation and the number of analytical methods applications for its solution is very limited. For the numerical calculation of the magnetic vortices bound dynamics a software package for micromagnetic simulations SpinPM was used.

Let us consider a nanopillar of circular cross-section with a diameter of $120 \mathrm{~nm}$ (Fig. 1). It contains three layers: thick magnetic layer of permalloy (15 nm thick), an intermediate magnetic layer (10 nm thick) and a thin magnetic layer of permalloy (thickness $4 \mathrm{~nm}$ ). The permalloy composition - $\mathrm{Ni}_{80} \mathrm{Fe}_{20}$, shorthand name - Py. System magnetic parameters are following: $M_{s}=700 \mathrm{erg} / \mathrm{Gs} \cdot \mathrm{cm}^{3}$ for a «thick» and $M_{s}=600 \mathrm{erg} / \mathrm{Gs} \cdot \mathrm{cm}^{3}$ for a «thin» layer, exchange rigidity $A=1.2 \cdot 10^{-6} \mathrm{erg} / \mathrm{cm}$ for a «thick» and $A=1.12 \cdot 10^{-6} \mathrm{erg} / \mathrm{cm}$ for a «thin» layer, $\alpha=0.01$, $\gamma=2.0023 \cdot 10^{7}(\mathrm{E} \cdot \mathrm{sec})^{-1}$. In these magnetic parameters, in absence of magnetic field and current, we have a quasihomogeneous configuration of magnetization in the thin magnetic layer and a vortex one in the thick layer [1]. To create a vortex state in a thin disk we use current. It is known that the switching of a spin-polarized current, through creating a circular Oersted field, can result in a vortex formation. By changing the current direction, the configurations from the parallel and antiparallel directed vortex core polarities can be obtained. The size of the cell for the calculations was $2 \times 2 \times 5 \mathrm{~nm}^{3}$.

We will investigate the dynamics of bound magnetic vortices with the same polarity and chirality. Current flows bottom up, perpendicularly to the layers plane, from a thick to thin layer. First let's take current polarization equal to $\mathrm{P}$ $=0.1$, the direction of the vortex cores polarity is upward. By using micromagnetic simulation, the dependence of the magnetic field size, switching the vortex core polarity in thin and thick layers, on current is found. (Fig. 2).

The simulations take into account the fact that both magnetic layers in the stack can act simultaneously as a spin polarizer and as a free layer when considering spin torque action. The current flow is assumed to be uniform through the pillar and the Oersted field is taken into account. A constant small in-plane field - 50 Oe is introduced to break the system's symmetry, but is not impacting the gyrotropic frequencies or any other dynamical properties. For each field and current value, we calculated the vortex dynamics of the nanopillar, with the initial state corresponding to the quiescent state at this field (i.e., with no current).

For the case of low currents, the vortex polarity switch in thin and thick layers was observed with a low exit of the vortex from the geometric center. Thus the vortex polarity switching mechanism is similar to «static», but with an excitation of vortex oscillations internal modes and spin waves radiation. Note that this scenario of the vortex core polarity switching, accompanied by the excitation of internal modes oscillations and spin waves radiation, was also observed in a single vortex case (see. e.g. [9]). For the case of high currents, the «dynamic» mechanism for the vortex core polarity switching [6] was observed only for a vortex in a thick layer. Let's compare the simulation and experimental results for the structure presented in [6]. For the case of a vortex in a thick layer, two currents region can be distinguished in the experimental curve - a region of low current and a high current region. Note that the position and size of this region in the experimental and theoretical cases are close to each other. Just as in the experimental graph, the critical magnetic field values of the theoretical graph are qualitatively the same in size and decrease with the increase of the current value. For the case of a vortex in a thin layer, in contrast to the experimental curve, where there are two current regions, we have for both low and high current a quasi-static vortex polarity switching mechanism.

To study the effect of the polarization current value, let us consider the case with $P=0.13$. From Fig. 3 we can see that similarly to the case with $R=0.1$ there are two regions of low and high currents.

For the region of high current, depending on the magnetic field value, for a vortex in a thick layer one can observe both dynamic and quasi-static mechanism of vortex polarity switching. The minimum current value in the high current region (similarly to the case of nanocylinder with a diameter of $200 \mathrm{~nm}$ [8]) significantly decreases with the increase of the current polarization value.

Acknowledgements. The authors are deeply indebted to K. Zvezdin both for his comments and for helpful discussions. This study was supported by Russian Foundation for Basic Research, project 16-32-00381.

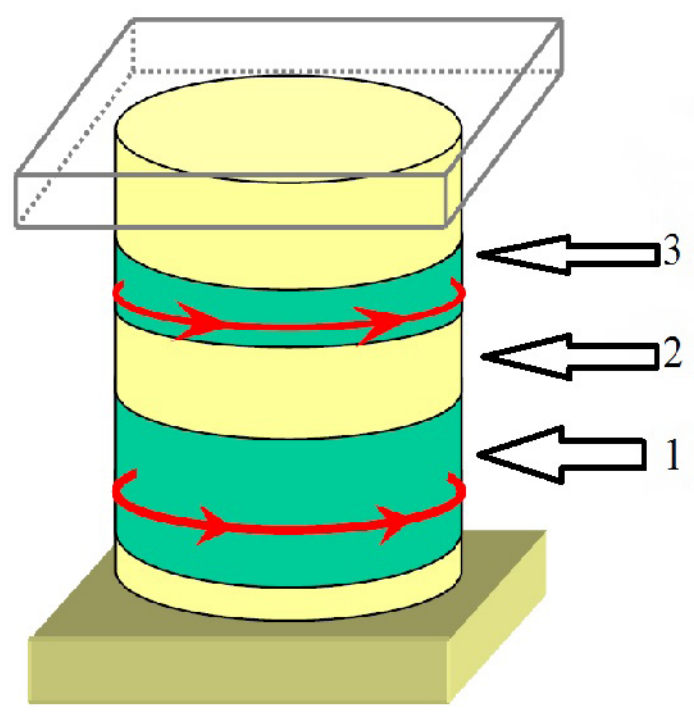

Fig. 1. Schematic representation of multi-layer nano-columnar structure.1- thick magnetic layer, 2 - non-magnetic layer, 3-thin magnetic layer. 


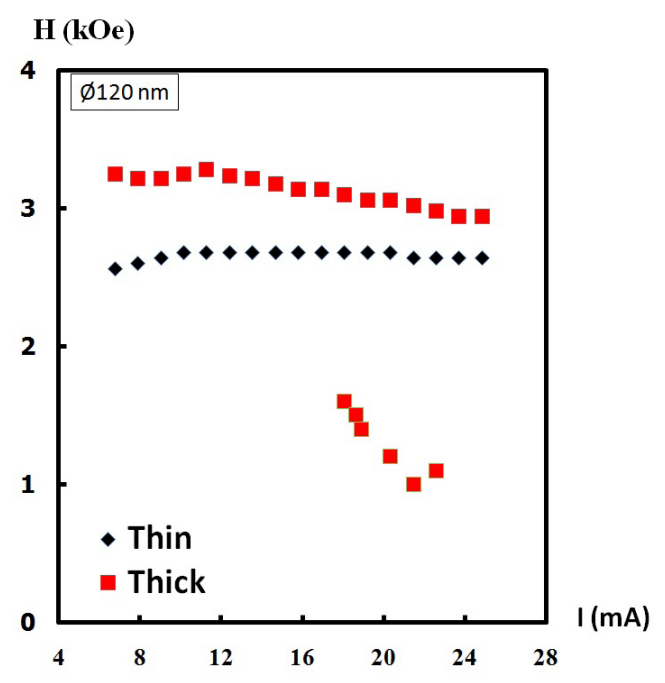

Fig. 2. Switching fields of the thin layer (diamonds) and the thick layer (squares) vortex cores for the $120 \mathrm{~nm}$ pillar, $P=0.1$, obtained from micromagnetic simulations.

\section{References}

1. K.Yu. Guslienko, J. Nanosc. Nanotechn. 8, 2745 (2008).

2. Y. Gaididei, V. P. Kravchuk, D. D. Sheka International Journal of Quantum Chemistry 110(1), 83, (2010).

3. S. Petit-Watelot, J. Kim, A. Ruotolo, R. M. Otxoa, K. Bouzehouane, J. Grollier, A. Vansteenkiste, B.V. d. Wiele, V. Cros, T. Devolder Nature Physics 8, 682 (2012).

4. T. Okuno, K. Shigeto, T. Ono, K. Mibu, and T. Shinjo. JMMM 240, 1 (2002).

5. A. V. Khvalkovskiy, A. N. Slavin, J. Grollier, K. A. Zvezdin, K. Y. Guslienko. Appl. Phys. Lett. 96, 022504 (2010).

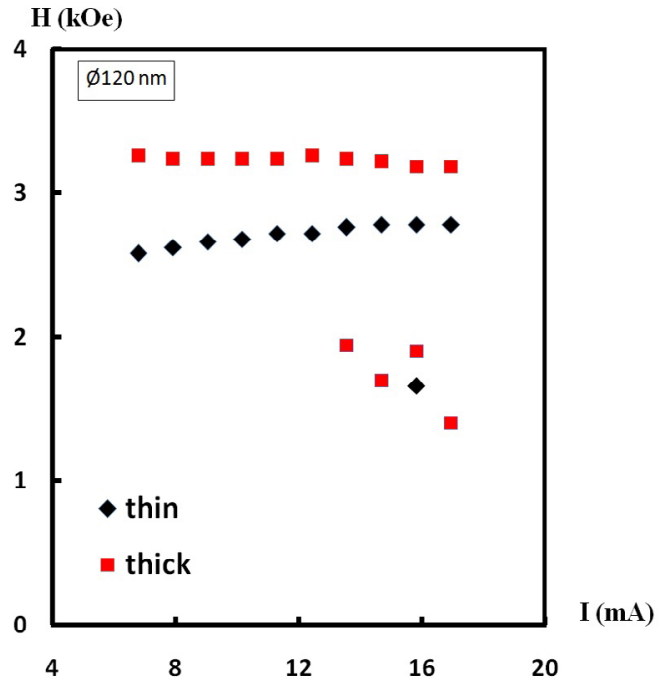

Fig. 3. Switching fields of the thin layer (diamonds) and the thick layer (squares) vortex cores for the $120 \mathrm{~nm}$ pillar, $P=0.13$, obtained from micromagnetic simulations.

6. N. Locatelli, A.E. Ekomasov, A.V. Khvalkovskiy, Sh.A. Azamatov, K.A. Zvezdin, J. Grollier, E.G. Ekomasov, V. Cros. Appl. Phys. Lett. 102, 062401 (2013).

7. N. Locatelli, R. Lebrun, V.V. Naletov, A. Hamadeh, G. De Loubens, O. Klein, J. Grollier, V. Cros IEEE Transactions on Magnetics 51, 8, 4300206 (2015).

8. A.E. Ekomasov, A.V. Khvalkovskiy, K.A. Zvezdin, E.G. Ekomasov. Bulletin of the Russian Academy of Sciences. Physics. 77, 1490 (2013) (in Russian).

9. Myoung-Woo Yoo, Jehyun Lee, and Sang-Koog Kim. Appl. Phys. Lett. 100, 172413 (2012). 